\title{
Avaliação de indicadores antropométricos de obesidade e a presença de comorbidades em participantes das ações do NASF do município de Candói, Paraná, Brasil
}

\author{
Evaluation of anthropometric indicators of obesity and the presence of comorbidities in \\ participants of NASF actions in the city of Candói, Paraná, Brazil
}

\author{
Fernando Juraski Lefchak ${ }^{1}$, Guilherme Welter Wendt ${ }^{2}$, Geraldo Emílio Vicentini ${ }^{3}$, Claudiceia Risso \\ Pascotto $^{4^{*}}$
}

${ }^{1}$ Mestre em Ciências Aplicadas à Saúde, Universidade Estadual do Oeste do Paraná (Unioeste); ${ }^{2}$ Doutor em Psicologia pela Universidade de Londres, Docente do Centro de Ciências da Saúde da Unioeste; ${ }^{3}$ Doutor em Ciências Farmacêuticas, Docente do Centro de Ciências da Saúde da Unioeste; ${ }^{4}$ Doutora em Biologia Celular, Docente do Centro de Ciências da Saúde da Unioeste

\begin{abstract}
Resumo
Introdução: alguns indicadores antropométricos se caracterizam por serem facilmente aplicados e amplamente utilizados para a avaliação da obesidade e distribuição da gordura corporal, constituindo um dos primeiros passos para identificar risco inicial à saúde. Objetivo: avaliar e identificar a frequência de obesidade geral e abdominal por meio de indicadores antropométricos e verificar a presença de comorbidades em indivíduos que participaram das reuniões educativas e atividades do NASF do Município de Candói, Paraná. Metodologia: trata-se de um estudo quantitativo e transversal, com aplicação de questionário para conhecer as comorbidades e caracterizar a amostra por meio da aferição de peso, estatura e circunferência da cintura (CC), além da determinação do índice de massa corporal (IMC) e relação cintura-estatura (RCE). Nas análises de associação, utilizou-se o teste de qui-quadrado seguido do cálculo das razões de chance (RC). Resultados: a maior parte da amostra foi classificada como sobrepeso/obesidade (69,2\%), com base no IMC, e apresentou elevados índices de obesidade abdominal pela CC e RCE. Os valores de CC aumentados foram observados em $83,01 \%$ dos pacientes. Em relação a RCE, o valor adequado foi verificado em apenas $14,5 \%$ dos participantes. As associações entre os índices antropométricos e as demais variáveis mostraram diferenças estatisticamente significativas em relação à idade dos participantes, revelando maior frequência de RCE aumentada em pacientes com 60 anos ou mais $(R C=8,53, p=0,01)$. Dentre as comorbidades, o destaque foi a presença da hipertensão arterial sistêmica. Conclusão: além das elevadas frequências de obesidade geral, obesidade abdominal e da presença de comorbidades, como a hipertensão arterial sistêmica, a RCE foi o índice antropométrico capaz de sugerir risco cardiometabólico aumentado em idosos. Neste sentido, este estudo mostra a capacidade de caracterizar os usuários do sistema público de saúde com potencial para o desenvolvimento de doença cardiovascular através do uso de ferramentas antropométricas de baixo custo, e assim, poder dar suporte para ações corretivas a nível de políticas públicas municipais.

Palavras-Chave: Obesidade. Circunferência da Cintura. Índice de Massa Corporal. Antropometria.
\end{abstract}

\begin{abstract}
Introduction: some anthropometric indicators are characterized by being easily applied and widely used to assess obesity and body fat distribution, constituting one of the first steps to identify initial health risk. Objective: to assess and identify the frequency of general and abdominal obesity using anthropometric indicators and to verify the presence of comorbidities in individuals who participated in the NASF educational meetings and activities in the city of Candói, Paraná. Methodology: this is a quantitative and cross-sectional study, with the application of a questionnaire to find out about comorbidities and to characterize the sample by measuring weight, height and waist circumference (WC), in addition to determining the body mass index (BMI) and waist-height ratio (RCE). In the association analyzes, the chi-square test was used followed by the calculation of the odds ratios (CR). Results: most of the sample was classified as overweight/obesity (69.2\%), based on BMI, and showed high rates of abdominal obesity by WC and WHR. The increased WC values were observed in $83.01 \%$ of the patients. Regarding CER, the adequate value was verified in only $14.5 \%$ of the participants. The associations between the anthropometric indices and the other variables showed statistically significant differences in relation to the age of the participants, revealing a higher frequency of increased CER in patients aged 60 years or older ( $R C=8.53$, $p=0.01$. Among the comorbidities, the highlight was the presence of systemic arterial hypertension. Conclusion: in addition to the high frequencies of general obesity, abdominal obesity and the presence of comorbidities, such as systemic arterial hypertension, the CER was the anthropometric index capable of suggesting increased cardiometabolic risk in the elderly. In this sense, this study shows the ability to characterize users of the public health system with the potential for the development of cardiovascular disease through the use of low-cost anthropometric tools, and thus be able to support corrective actions at the level of municipal public policies.
\end{abstract}

Correspondente/Corresponding: *Claudicéia Risso Pascotto-End: Centro de Ciências da Saúde - Rodovia PR 182 Vitório Traiano, s/n - Contorno Leste, Bairro Água Branca, CEP: 85601-970, Francisco Beltrão-PR. - Tel: (46) 3520-0816/99982-1110-E-mail: claudiceia.pascotto@unioeste.br
Keywords: Obesity. Waist circumference. Body mass index. Anthropometry. 


\section{INTRODUÇÃO}

A análise da distribuição de gordura na prática clínica ainda permanece um desafio. Apesar dos métodos de imagens oferecerem uma melhor sensibilidade para medir os depósitos específicos de gordura, os índices antropométricos são os mais utilizados por serem considerados de fácil implementação (NEELAND et al., 2019). As avaliações antropométricas podem ser consideradas como um dos primeiros passos para identificar indivíduos com risco inicial à saúde, apresentando utilidade ao indicar para uma triagem mais aprofundada quando seus valores se apresentam acima dos limites (ASHWELL; GIBSON, 2016).

A International Atherosclerosis Society (IAS) e a International Chair on Cardiometabolic Risk (ICCR) reconhecem que médicos e pesquisadores devem transmitir uma mensagem de saúde pública para que haja maior atenção à gordura visceral, além do excesso de peso, com o intuito de auxiliar a combater a epidemia causada no mundo pela obesidade (NEELAND et al., 2019).

A obesidade é considerada uma condição multifatorial e evitável, definida pelo excesso de gordura corporal. Habitualmente, é estimada pela razão entre o peso e a estatura, o chamado índice de massa corporal (IMC) e pode indicar risco aumentado para o desenvolvimento de comorbidades caso seus índices estejam fora dos valores ideais (NATIONAL INSITUTE FOR HEALTH AND CARE EXCELLENCE, 2014).

Além do IMC, autores apoiam a ideia de que os profissionais devem inserir outros indicadores que avaliem a distribuição da gordura corporal (ASHWELL; GIBSON, 2016). Dentre eles, a circunferência da cintura (CC) e a relação cintura/estatura (RCE), os quais são de fácil aplicabilidade (ABESO, 2016) e têm a vantagem de serem métodos clínicos sem o uso de imagem (NEELAND et al., 2019).

A CC é um indicador bastante utilizado para a aferição do tecido adiposo central. É mensurada através de avaliações individuais e coletivas, e quando aumentado, é preditivo da presença de elevada gordura abdominal (HAUN; PITANGA; LESSA, 2009). Esta circunferência apresenta associação positiva com maiores níveis de mortalidade, podendo ser utilizada em todos os níveis de IMC, desde obesos a não obesos (CERHAN et al., 2014). Já a RCE indica o nível de obesidade concentrada na região abdominal e tem forte associação com diversos fatores de risco cardiometabólicos, o que sugere seu uso em estudos populacionais (PITANGA; LESSA, 2005).

Há fortes evidências de que o risco de doença cardiovascular está mais relacionado à forma corporal e à distribuição do tecido adiposo, o qual pode ser mensurado pela CC e RCE, do que ao IMC ou a um excesso de gordura corporal total (DESPRÉS, 2012). Sendo estes índices de obesidade central adequados para o emprego em triagem primária e em programas de promoção da saúde e prevenção de doenças (ASHWEEL; GIBSON,
2016). Os índices de excesso de peso e obesidade vêm crescendo em vários países nos últimos anos (NATIONAL INSITUTE FOR HEALTH AND CARE EXCELLENCE, 2014). No Brasil, um estudo com a população adulta, realizado em 26 capitais e no distrito federal, identificou $54 \%$ de excesso de peso e 18,9\% de obesidade (BRASIL, 2018). De acordo com Malta, Morais Neto e Silva Junior (2011), manter dados atualizados sobre a distribuição, evolução e frequência desses indicadores no Brasil é fundamental para o monitoramento de metas previstas pelo Plano de Ações Estratégicas para o Enfrentamento das Doenças Crônicas não Transmissíveis (DCNT).

Desta forma, o Ministério da Saúde recomenda que os profissionais do Sistema Único de Saúde (SUS) inseridos no Núcleo de Apoio à Saúde da Família (NASF), incluam na atenção básica indicadores antropométricos para avaliação e identificação do excesso e distribuição da gordura corporal como forma de rastreamento individual e coletivo no diagnóstico e classificação de riscos à saúde, visando contribuir com planejamento e ações eficazes de acordo com as características de cada população específica (BRASIL, 2017). Assim, este estudo teve como objetivo identificar a frequência de obesidade geral e abdominal por meio de indicadores antropométricos e a presença de comorbidades em indivíduos que participaram das reuniões educativas e atividades do NASF do Município de Candói, Paraná.

\section{METODOLOGIA}

Este estudo caracteriza-se como de delineamento quantitativo e transversal. A amostra selecionada por conveniência foi composta por 159 pacientes, de um total de 479 que estiveram presentes durante as reuniões educativas e atividades do NASF do Município de Candói (Paraná, Brasil), nos meses de agosto a dezembro do ano de 2018. Os pacientes foram abordados durante a atividade da equipe e convidados para responder ao questionário e participar da avaliação antropométrica. Deste modo, todos os inclusos concordaram em participar da pesquisa e assinaram o Termo de Consentimento Livre e Esclarecido (TCLE).

Não foram incluídos os seguintes pacientes: que não sabiam ler ou escrever, cadeirantes ou portadores de necessidades especiais, gestantes, pessoas que informaram ser portadores de doenças graves como HIV/AIDS e/ou câncer, e ainda, indivíduos que haviam realizado algum tipo de cirurgia nos últimos seis meses que pudesse comprometer significativamente a movimentação e funcionalidade corporal.

Esta pesquisa está em conformidade com a Resolução n.o 196/96 do Conselho Nacional de Saúde e seus complementos e foi aprovada pelo Comitê de Ética em Pesquisa com Seres Humanos da Universidade Estadual do Oeste do Paraná - Unioeste, conforme parecer número 2.787.844. 
Para caracterização da amostra, foi aplicado um questionário contendo as variáveis: idade, sexo, estado civil, número de filhos, escolaridade, tipo de domicílio e o número de residentes no mesmo domicílio, renda e tipo de esgotamento sanitário e a presença de comorbidades. Foram aferidas as medidas antropométricas de massa corporal, estatura e circunferência da cintura. Tais medidas foram tomadas três vezes e o valor utilizado nesse estudo corresponde à média aritmética simples (WANG et al., 2003). Na avaliação da massa corporal, foi registrado o valor em quilogramas $(\mathrm{kg})$, e utilizou-se uma balança digital (803 SECA) com precisão de 100 gramas, posicionada em superfícies regulares e firmes. Os pacientes estavam vestindo roupas leves, com os bolsos vazios e sem acessórios, descalços e com os dois pés apoiados na plataforma e olhando para a linha do horizonte (CENTER FOR DISEASE CONTROL, 2009). A estatura foi aferida em metros $(m)$, utilizando um estadiômetro portátil adaptável (217 SECA) com precisão de um milímetro, posicionado em paredes sem rodapé e pisos regulares. Os participantes ficaram descalços, em pé, com as pernas e pés paralelos, braços relaxados ao lado do corpo e palmas das mãos voltadas para o tronco, com a coluna direcionada para o aparelho, calcanhares, panturrilhas, nádegas, escápulas e parte posterior do osso occipital encostados no equipamento e, caso não fosse possível os cinco pontos, foram posicionados ao menos três deles (calcanhares, glúteos e costas), com a cabeça sem adereços, no plano de Frankfurt (CENTER FOR DISEASE CONTROL, 2009). Estes dados foram utilizados para posterior cálculo do IMC em kg/m2 (razão entre o peso atual em $\mathrm{kg}$ sobre o quadrado da estatura em $\mathrm{m}^{2}$ ). A classificação utilizada para o IMC foi: baixo peso para valores de IMC $<18,5 \mathrm{~kg} / \mathrm{m}^{2}$; normal ou eutrófico entre $18,5 \mathrm{~kg} / \mathrm{m}^{2}$ até $24,9 \mathrm{~kg} / \mathrm{m}^{2}$; sobrepeso de $25,0 \mathrm{~kg}$ / $\mathrm{m}^{2}$ a $29,9 \mathrm{~kg} / \mathrm{m}^{2}$; e obeso $\geq 30,0 \mathrm{~kg} / \mathrm{m}^{2}$ (ABESO, 2016; WORLD HEALTH ORGANIZATION, 2000).

A CC foi avaliada por meio de uma fita métrica no ponto médio entre a última costela e a crista ilíaca, no momento final da expiração normal (ABESO, 2016). Para homens, o ponto de corte adotado foi de 90 centímetros (cm) e, para mulheres, de $80 \mathrm{~cm}$ (ALBERTI; ZIMMET; SHAW, 2006). Para a RCE, foi realizada a divisão da medida da cintura pela estatura, ambas em centímetros, utilizando o ponto de corte 0,5 para homens e mulheres. A RCE e a CC são bons preditores de risco cardiometabólico, ou seja, prediz fatores de risco para doença cardiovascular e diabetes (BROWNING; HSIEH; ASHWELL, 2010).

Os dados foram tabulados utilizando o programa Microsoft Office Excel 2016, os quais foram inspecionados quanto à presença de erros de digitação e omissão e possíveis casos extremos (outliers). Com o propósito de descrever as principais variáveis, utilizou-se de procedimentos de estatística descritiva, reportando valores mínimos, máximos, médias, desvios-padrão (DP) e porcentagens. Para as análises de associação entre presença de comorbidades (sim/não), sexo, idade (adultos/idosos), relação cintura/estatura (adequada/ aumentada), circunferência da cintura (adequada/ aumentada) e IMC (baixo peso, eutrófico, sobrepeso e obesidade), utilizou-se o teste de qui-quadrado com correção de continuidade de Yates ou Teste Exato de Fisher, seguidos da análise de razões de chance (RC), apresentado com intervalo de confiança (IC) de 95\%. Tais análises descritivas e inferenciais foram realizadas no programa SPSS, versão 23.

\section{RESULTADOS}

A média de idade da amostra foi de 48,57 anos ( $D P=14,55)$, predominantemente composta pelo sexo feminino $82,39 \%(n=131)$, e $17,61 \%(n=28)$ masculino. Quanto ao estado civil, a frequência de casados foi de $61,01 \%(n=97)$, de solteiros, $11,95 \%(n=19)$, em união estável, 15,09\% ( $n=24)$, divorciados, $2,52 \%(n=4)$ e viúvos, 9,43\% ( $n=15)$. A média do número de filhos foi de $2,79$, em que a maioria, $22,64 \%$ ( $n=36)$, afirmou ter cinco ou mais, $11,32 \%$ ( $n=18)$, quatro filhos, $20,75 \%(n=33)$, três, $21,38 \%(n=34)$, dois, $15,72 \%(n=25)$, um, e $8,18 \%$ $(n=13)$ nenhum filho.

Em relação ao tipo de domicílio, $84,28 \%(n=134)$ residiam em casa própria, $6,29 \%(n=10)$ moravam de aluguel, e 9,43\% ( $n=15)$ em imóvel cedido pelo empregador. Quanto ao quantitativo de moradores por domicílio, a média foi de três pessoas, dos quais $6,92 \%(n=11)$ moravam sozinhos, $30,19 \%(n=48)$ em dois moradores, $31,45 \%(n=50)$ em três, $18,87 \%(n=30)$ quatro e $12,58 \%$ $(n=20)$ com cinco ou mais. As formas de saneamento utilizada foram através da rede geral de esgoto ou galeria pluvial, $24,53 \%$ ( $n=39)$, por fossa séptica, $44,65 \%$ $(n=71)$, fossa rudimentar, $29,56 \%(n=47)$, rio ou lago, $0,63 \%(n=1)$, e de outra forma, $0,63 \%(n=1)$.

Sobre a renda familiar mensal, $30,19 \%(n=48)$ responderam ser menor que um salário mínimo, 55,35\% $(n=88)$ de um até dois, $10,06 \%(n=16)$ de dois até quatro, $2,52 \%(n=4)$ de quatro até seis, $0,63 \%(n=1)$ de seis até oito, $0,63 \%(n=1)$ de oito até dez e $0,63 \%(n=1)$ superior a dez salários mínimos. Vale ressaltar que os valores foram referentes ao salário mínimo nacional vigente no período da pesquisa, no valor de $\mathrm{R} \$ 954,00$ (novecentos e cinquenta e quatro reais).

Quando se trata de escolaridade, $11,32 \%$ ( $n=18)$ não frequentou o ensino regular, 54,72\% $(n=87)$ assinalou como ensino fundamental incompleto, $10,06 \%(n=16)$ apresentam o ensino fundamental completo, 5,03\% $(n=8)$ ensino médio incompleto, $16,35 \%(n=26)$ ensino médio completo, $0,63 \%(n=1)$ ensino superior incompleto, e apenas $1,89 \%(n=3)$ com ensino superior completo ou pós-graduação.

Os resultados da avaliação antropométrica com massa corporal, estatura, valores do IMC e CC estão apresentados na tabela 1. Primeiramente de forma isolada, e na sequência através do parâmetro da relação cintura-estatura. 
Tabela 1 - Caracterização geral das variáveis antropométricas.

\begin{tabular}{lrrrr}
\hline & Média & Desvio-padrão & Mínimo & Máximo \\
\hline Massa Corporal (Kg) & 72,23 & 17 & 39,5 & 140,9 \\
Estatura (m) & 1,59 & 0,80 & 1,41 & 1,85 \\
CC (cm) & 97,49 & 15,61 & 63,00 & 160,00 \\
IMC (kg/m²) & 28,45 & 6,24 & 17,30 & 51,20 \\
RCE $(\mathrm{cm})$ & 0,61 & 0,10 & 0,42 & 0,96 \\
\hline
\end{tabular}

${ }^{*} C C$ - circunferência da cintura; IMC - índice de massa corporal; RCE relação cintura/estatura.

Fonte: Dados da pesquisa

Após a classificação pelo IMC, foi verificado que dois participantes $(1,3 \%)$ atenderam aos critérios de baixo peso, $47(29,6 \%)$ estavam eutróficos, $59(37,1 \%)$ sobrepeso e $51(32,1 \%)$ com obesidade. Ou seja, $69,2 \%$ da amostra apresentou excesso de peso (IMC $\left.\geq 25 \mathrm{~kg} / \mathrm{m}^{2}\right)$.

Analisando a variável $\mathrm{CC}$, valores acima do ponto de corte $(\geq 80 \mathrm{~cm})$ foram encontrados em 112 mulheres $(85,5 \%)$. Em relação aos homens, 20 (71,4\%) estavam acima do ponto de corte $(\geq 90 \mathrm{~cm})$. Assim, observamos que 132 pacientes apresentaram valores de CC aumentados, o que representa $83,01 \%$ da amostra. Para a variável RCE, o valor adequado foi verificado em 23 (14,5\%) participantes. Logo, $136(85,5 \%)$ apresentaram valores acima do ponto de corte.

Em relação às comorbidades autorreferidas, a hipertensão arterial sistêmica (HAS) foi a mais relatada. Ela esteve presente em 57 (35,84\%) dos 159 entrevistados, tanto de forma isolada quanto em combinação com outras comorbidades, como pode ser observado na tabela 2. A segunda mais relata foi $D M 2$ em $6,91 \%(n=11)$ dos casos, seguida de DM1 (5,03\%) e depressão (5,03\%), que ocupam o terceiro lugar, sendo citadas por 8 pessoas. As combinações e demais comorbidades autorreferidas estão listadas na tabela 2 . Nota-se ainda que $13,21 \%$ relataram não possuir nenhuma comorbidade ( $n=21), 8,81 \%$ não souberam ( $n=14)$, e $27,04 \%(n=43)$ não quiseram informar.
Tabela 2 - Comorbidades autorreferidas pelos entrevistados.

\begin{tabular}{lcc}
\hline & $\begin{array}{c}\text { Frequência } \\
\text { (n) }\end{array}$ & $\begin{array}{c}\text { Percentual } \\
(\%)\end{array}$ \\
\hline HAS & 31 & 19,50 \\
HAS e DM2 & 6 & 3,77 \\
HAS e DM1 & 7 & 4,40 \\
HAS e depressão & 9 & 5,66 \\
HAS, DM1 e depressão & 1 & 0,63 \\
HAS e neurocisticercose & 1 & 0,63 \\
HAS e osteoporose & 1 & 0,63 \\
HAS e bursite & 1 & 0,63 \\
DM 2 & 5 & 3,14 \\
Ansiedade & 1 & 0,63 \\
Artrite e artrose & 1 & 0,63 \\
Bronquite asmática & 1 & 0,63 \\
Cefaleia & 1 & 0,63 \\
Depressão & 7 & 4,40 \\
Depressão e hipertireoidismo & 1 & 0,63 \\
Espondilolistese & 1 & 0,63 \\
Hepatite B & 1 & 0,63 \\
Hipertireoidismo & 3 & 1,89 \\
Rinite e psoríase & 1 & 0,63 \\
Sem comorbidades & 21 & 13,21 \\
Não sabiam informar & 14 & 8,81 \\
Não quiseram informar & 43 & 27,04 \\
Outro & 1 & 0,63 \\
\hline
\end{tabular}

*DM1 - Diabetes Mellitus Tipo 1; DM2 - Diabetes Mellitus Tipo 2; HAS - Hipertensão Arterial Sistêmica.

Fonte: Dados da pesquisa

A Tabela 3 apresenta as comparações entre IMC, CC e RCE em relação ao sexo, idade e ocorrência de comorbidades na amostra. Diferenças estatisticamente significativas foram encontradas em relação à RCE, em que participantes com mais de 60 anos tiveram uma razão de chance 8,53 (IC 95\%: 1,11-65,52, $p=0,01$ ) vezes maior de apresentar uma RCE elevada em comparação com os participantes mais jovens.

Tabela 3 - Comparações entre IMC, CC e RCE em relação ao sexo, idade e ocorrência de comorbidades na amostra.

\begin{tabular}{|c|c|c|c|c|c|c|}
\hline \multirow[b]{2}{*}{ Variáveis } & \multicolumn{2}{|c|}{ Sexo } & \multicolumn{2}{|c|}{ Patologias } & \multicolumn{2}{|c|}{ Faixa etária } \\
\hline & $\begin{array}{c}\text { Masculino } \\
\text { n (\%) }\end{array}$ & $\begin{array}{c}\text { Feminino } \\
\mathrm{n}(\%)\end{array}$ & $\begin{array}{l}\text { Não } \\
\text { n (\%) }\end{array}$ & $\underset{n(\%)}{\operatorname{Sim}}$ & $\begin{array}{c}\leq 59 \text { anos } \\
n(\%)\end{array}$ & $\begin{array}{c}\geq 60 \text { anos } \\
n(\%)\end{array}$ \\
\hline \multicolumn{7}{|l|}{$\mathrm{CC}$} \\
\hline adequada & $8(28,6 \%)$ & $19(14,5 \%)$ & $6(28,6 \%)$ & $11(13,6 \%)$ & $24(20,0 \%)$ & $3(7,7 \%)$ \\
\hline aumentada & $20(71,4 \%)$ & $112(85,5 \%)$ & $15(71,4 \%)$ & $70(86,4 \%)$ & $96(80,0 \%)$ & $36(92,3 \%)$ \\
\hline RC (IC 95\%) & & $2,36(0,90-6,12)^{1}$ & & $2,55(0,81-7,96)^{1}$ & & $3,00(0,85-10,6)^{2}$ \\
\hline \multicolumn{7}{|l|}{ RCE } \\
\hline adequada & $4(14,3 \%)$ & $19(14,5 \%)$ & $5(23,8 \%)$ & $10(12,3 \%)$ & $22(18,3 \%)$ & $1(2,6 \%)$ \\
\hline aumentada & $24(85,7 \%)$ & $112(85,5 \%)$ & $16(76,2 \%)$ & $71(87,7 \%)$ & $98(81,7 \%)$ & $38(97,4 \%)$ \\
\hline RC (IC 95\%) & & $0,98(0,30-3,15)^{2}$ & & $2,22(0,66-7,39)^{1}$ & & $8,53(1,11-65,5)^{2}$ \\
\hline \multicolumn{7}{|l|}{ IMC } \\
\hline Baixo peso ou Eutrófico & $11(39,3 \%)$ & $38(29,0 \%)$ & $8(38,1 \%)$ & $23(28,4 \%)$ & $41(34,2 \%)$ & $8(20,5 \%)$ \\
\hline Sobrepeso e obesidade & $17(60,7 \%)$ & $93(71,0 \%)$ & $13(61,9 \%)$ & $58(71,6 \%)$ & $79(65,8 \%)$ & $31(79,5 \%)$ \\
\hline RC (IC 95\%) & & $1.58(0,67-3,70)^{1}$ & & $1.55(0,56-4,24)^{1}$ & & $2.01(0,84-4,77)^{1}$ \\
\hline
\end{tabular}

*CC - circunferência da cintura; IMC - índice de massa corporal; RC-razão de chance (odds ratio); e IC-intervalo de confiança de $95 \%$; $R C E$ - razão cintura/estatura. ${ }^{1}$ Teste de qui-quadrado com correção de continuidade de Yates; ${ }^{2}$ Teste Exato de Fisher.

Fonte: Dados da pesquisa 


\section{DISCUSSÃO}

O monitoramento de indicadores de prevalência de obesidade é extremamente necessário para buscar entender os padrões de risco da população com maior vulnerabilidade no país. Ele visa subsidiar políticas em saúde pública para a prevenção de sobrepeso e obesidade e a promoção de hábitos saudáveis no Brasil (FERREIRA et al., 2018).

Neste sentido, o presente estudo reporta achados acerca da presença de obesidade geral e abdominal e a presença de comorbidades em participantes das ações do NASF do município de Candói - Paraná, de um grupo de adultos de meia idade, com grande percentual de mulheres. A maior parte foi de indivíduos casados, com filhos, que residiam em domicílio próprio, caracterizados por possuir baixa renda e pouca escolaridade, e que apresentaram valores aumentados para os índices antropométricos utilizados, tanto para a obesidade geral quanto para a abdominal.

Historicamente, a procura pelos serviços de saúde tem demonstrado predominância das mulheres, sendo que estas buscam o serviço de saúde cerca de 1,9 vezes mais que os homens (LEVORATO et al., 2014). A última Pesquisa Nacional de Saúde (PNS) realizada no Brasil em 2013, em uma parceria do Instituto Brasileiro de Geografia e Estatística (IBGE) e o Ministério da Saúde sobre o acesso e a utilização dos serviços de saúde, acidentes e violência, identificou que a proporção de procura por atendimentos de saúde foi maior pelas mulheres, representando 18,5\% contra 11,9\% dos homens (IBGE, 2015).

Em pesquisa realizada com 1433 indivíduos em uma área rural de Pelotas, encontrou-se idade média similar a dos participantes do presente artigo (MARTINS-SILVA et al., 2018). Quanto à escolaridade, identificaram que a maior porcentagem foi baixa (entre zero e quatro anos de estudo), e quanto ao estado conjugal a grande maioria, assim como em nosso estudo, também viviam com companheiro. Os autores realizaram ainda a associação entre a escolaridade e obesidade e demonstraram que as mulheres com mais anos de estudo apresentaram proteção em relação a obesidade geral e abdominal (MARTINS-SILVA et al., 2018).

Essa relação do IMC com a escolaridade e a idade é demonstrada recentemente pelo Ministério da Saúde no Brasil. Onde a frequência do excesso de peso (IMC $\geq$ $25 \mathrm{~kg} / \mathrm{m}^{2}$ ) entre as mulheres apresentou uma tendência de aumento devido à idade mais elevada. Entretanto, houve a diminuição dos índices conforme maior escolaridade. Para a obesidade, especificamente em relação as mulheres, ficou demonstrado que a frequência diminui acentuadamente com maior nível de estudo. Tanto em mulheres quanto em homens com até 34 anos de idade, houve uma frequência menor de indivíduos obesos (IMC $\geq 30 \mathrm{~kg} / \mathrm{m}^{2}$ ) (BRASIL, 2018). Resultados similares foram encontrados por Martins-Silva et al. (2018), onde 29,5\% foram classificados com obesidade geral (IMC $\left.\geq 30 \mathrm{~kg} / \mathrm{m}^{2}\right)$, com valor médio muito próximo ao do presente estudo, estando em $27,7 \mathrm{~kg} / \mathrm{m}^{2}$. Além disso, Martins-Silva et al. (2018) analisaram os desfechos para a obesidade abdominal a partir da CC e um terceiro desfecho para avaliar o risco metabólico, revelando alta prevalência de obesidade geral e abdominal. Percentual elevado de excesso de peso também foi encontrado em outro estudo, em que $72,77 \%$ da amostra foi classificada pela composição corporal em situação acima da recomendada pela World Health Organizaton (DELBIM; BACIUK, 2016).

Na última PNS no Brasil, o excesso de peso dos participantes com base no IMC foi de $58,9 \%$ para o sexo feminino e $56,5 \%$ para o masculino. Já a obesidade, foi de $24,4 \%$ nas mulheres e de $16,8 \%$ nos homens (FERREIRA; SZWARCWALD; DAMACENA, 2019). Entretanto, quando as análises foram realizadas para verificar as associações entre o IMC e as demais variáveis de interesse, notou-se que não houve diferença entre homens e mulheres, entre jovens e idosos, assim como entre pacientes com e sem comorbidades. Estes dados podem ser úteis para reforçar o fato de Gibson e Ashwell (2015) apontarem implicações potencialmente graves na utilização do IMC isoladamente, uma vez que o seu uso não é adequado para a triagem de fatores de risco cardiometabólicos. Além disso, apesar da utilidade do IMC, a sua limitação está em não distinguir entre as massas gorda e magra. Neste sentido, a adiposidade abdominal, além da diminuição da massa muscular nos membros inferiores, é que estão mais associados a um maior nível de risco para o desenvolvimento de doença cardiovascular (POIRIER, 2015).

Em relação à obesidade abdominal, observamos, no presente trabalho, que a maior parte da amostra apresentou CC acima do ponto de corte utilizado. Valores de CC também foram identificados por Martins-Silva et al. (2018), em que o sexo feminino obteve um quadro mais elevado para a obesidade abdominal em comparação aos homens, o que corrobora com o presente artigo. A medida da CC é um importante parâmetro para avaliar a obesidade central e está relacionada com o risco metabólico (ABESO, 2016; ALBERTI; ZIMMET; SHAW, 2006). Investigações acerca da obesidade central podem ser utilizadas para que sejam implementadas estratégias de prevenção da população que apresente seus níveis elevados, representando assim uma classe importante para mudanças em relação ao estilo de vida (SAHAKYAN et al., 2015). A International Chair on Cardiometabolic Risk (ICCR) e a International Atherosclerosis Society (IAS), ressaltam que os profissionais da área da saúde devem utilizar a CC como um importante sinal vital na avaliação e identificação dos pacientes na prática clínica, e ainda, que precisam ser treinados com o intuito de medir essa circunferência adequadamente (ROSS et al., 2020).

Estudos realizados no Brasil demonstram que a utilização RCE nas avaliações antropométricas tem bons resultados na predição de risco cardiometabólico (CORRÊA et al., 2017; CORRÊA et al., 2019; MILAGRES et al., 2019; REIS et al., 2018; REZENDE et al., 2018). Para esta variável, 
no presente estudo, a maior parte da amostra apresentou valores acima do ponto de corte de 0,5 . Média próxima de RCE foi observada por Corrêa et al. (2017), com valor de 0,60 , sendo que a mesma apresentou satisfatório poder de detecção de excesso de peso. Entretanto, este resultado foi obtido ao avaliar apenas idosos, sendo indicada assim, pela sua simplicidade.

O estudo de Milagres et al. (2019) corrobora com nosso estudo ao reportar elevado percentual de RCE, em $88,1 \%$ da amostra. As médias foram de 0,56 para os homens e 0,63 para as mulheres. Seus resultados foram a partir de dados de 402 idosos atendidos na Estratégia de Saúde da Família, os quais permitiram demonstrar que homens e mulheres com RCE aumentado $(\geq 0,50)$ têm risco para alterações metabólicas, incluindo o percentual de gordura corporal (avaliada pela bioimpedância tetrapolar) e o excesso de peso (mensurado através do IMC). Reis et al. (2018), também reportaram que $80,0 \%$ da amostra apresentou RCE aumentada.

Cabe ressaltar que, de certo modo, é complexa a comparação entre os estudos abordando a RCE, pois fatores como faixa etária e diferentes pontos de corte variam entre os estudos (MILAGRES et al., 2019). Ashwell e Gibon (2016) propõe valores específicos em relação à esta variável, onde a classificação da RCE $\geq 0,5$ designa indivíduos com risco cardiometabólico aumentado, mesmo sem combinar os valores com o do IMC. Outro achado em relação à RCE na atual pesquisa concerne diferenças estatisticamente significativas em relação à idade dos participantes, sendo que, dentre os participantes com 60 anos ou mais, houve uma razão de chance de 8,53 para RCE alterada (IC 95\% 1,11-65,5) em comparação com aqueles com menos de 59 anos. Em seu estudo, Corrêa et al. (2019) encontraram diferenças importantes ao analisarem as médias da RCE conforme as classes de idade em adultos, tanto para os homens quanto para as mulheres. Já ao analisarem os idosos, os valores médios não apresentaram variação significativamente estatística. Estes dados podem corroborar para reforçar a utilização da RCE como preditor de excesso de peso para a população adulta (BROWNING; HSIEH; ASHWELL, 2010). Ainda, Ashwell e Gibson (2016) sugerem a utilização da razão entre a CC e a estatura como uma medida antropométrica que pode vir até a substituir a associação entre a CC e o IMC, tanto pela capacidade de identificação de indivíduos com risco à saúde, quanto pelo fato de estar associada a fatores de risco cardiometabólicos.

Corrêa et al. (2019) analisaram a relação entre RCE, risco à saúde e hipertensão em uma amostra de 8.235 adultos e 5.494 idosos, concluíram que a RCE foi superior em relação à combinação de IMC e CC para identificar indivíduos com risco precoce à saúde, além de estar associada à incidência de HAS, independentemente da faixa etária e do sexo. No estudo de Rezende et al. (2018), analisando 471 indivíduos com mais de 18 anos, determinou-se que a RCE, além do IMC e da CC, com significância estatística para o sexo feminino, esteve associada há um risco de incidência de hipertensão.

A presença de HAS, dentre as comorbidades, foi a mais presente em nosso estudo, afetando mais de $30 \%$ da amostra. Observou-se ainda a presença de DM2, DM1, depressão, dentre outras comorbidades autorreferidas. No estudo de Klauck et al. (2019) a HAS também foi a comorbidade mais diagnosticada, em $64,1 \%$ dos pacientes, já a DM1 esteve presente em $17,2 \%$. Em pesquisa nacional, a frequência de diagnóstico médico para a HAS foi de $24,3 \%$, enquanto a frequência de DM foi de $7,6 \%$ na população pesquisada (BRASIL, 2018). Pode-se perceber que tanto em estudos com maior número populacional, quanto em pesquisas através de amostras menores citados anteriormente, a presença de HAS e DM. Estes dados podem ser úteis para reforçar a gravidade do aumento desses problemas no campo da saúde pública no Brasil (FERREIRA et al., 2018).

Ainda, destaca-se um aspecto relacionado à informação, ou, neste caso, a desinformação, uma vez que $27,04 \%$ dos participantes não quiseram informar suas comorbidades e $8,81 \%$ não souberam informar. Acredita-se que tal fato não deixa de ser um achado importante, e que pode ser levado em consideração. Estes dados podem sugerir um possível desconhecimento ou falta de informação da amostra em relação ao seu estado de saúde e, também, uma possível negligência no momento do repasse das informações para a equipe de profissionais do NASF do município.

Constatou-se que dados epidemiológicos das regiões interioranas do país são ainda escassos. Portanto, depreende-se a necessidade da realização de pesquisas com o intuito de melhor conhecer as características específicas de usuários do sistema público de saúde, através do NASF, principalmente das regiões do interior do país.

Além disso, as comorbidades foram autorreferidas pelos pacientes. Salienta-se que, informações sobre comorbidades de forma autorreferida pelos participantes, podem introduzir vieses na compreensão da incidência de doenças. Além do mais, o estudo deve ser considerado em face de algumas limitações, como a adoção de delineamento transversal, o que impede uma compreensão mais apurada de relações de causa e efeito. Sugere-se que mais estudos através das medidas antropométricas sejam realizados, a fim de verificar o estado nutricional e identificar as comorbidades em usuários do SUS.

\section{CONCLUSÃO}

Através deste estudo, foram observadas frequências elevadas de obesidade geral (determinada através do IMC) e a presença de obesidade abdominal (avaliada pela (C). Além disso, a maior parte da amostra apresentou o indicador RCE aumentado. Através das alterações da CC e da RCE demonstradas nesta pesquisa, pode-se inferir, portanto, risco de complicações cardiometabólicas. Ademais, os pacientes relataram a presença de 
diversas comorbidades, dentre as quais, a mais presente foi a hipertensão arterial sistêmica. Em suma, o estudo demonstra a capacidade de caracterizar os usuários do sistema público de saúde com potencial aumentado para o desenvolvimento de doença cardiovascular através da utilização de indicadores antropométricos, considerados de baixo custo e fácil acesso. Assim, podem dar suporte para que sejam realizadas, no futuro, ações corretivas e/ ou preventivas ao nível de políticas públicas municipais relacionadas à saúde.

\section{AGRADECIMENTOS}

Aos coordenadores dos setores públicos de saúde do município de Candói, Paraná, pela autorização da coleta de dados.

\section{REFERÊNCIAS}

ASSOCIAÇÃO BRASILEIRA PARA O ESTUDO DA OBESIDADE E DA SÍNDROME METABÓLICA (ABESO). Diretrizes brasileiras de obesidade. 4. ed. São Paulo, 2016. P. 7-186. Disponível em: https://abeso.org.br/wpcontent/uploads/2019/12/Diretrizes-Download-Diretrizes-Brasileirasde-Obesidade-2016.pdf. Acesso em: 25 Mar. 2020.

ALBERTI, K. G. M. M.; ZIMMET, P.; SHAW, J. Metabolic syndrome-A new world-wide definition. A Consensus Statement from the International Diabetes Federation. Diabet. Med., Chichester, v. 23, n. 5, p. 469-480, 2006.

ASHWELL, M.; GIBSON, S. Waist-to-height ratio as an indicator of 'early health risk': simpler and more predictive than using a 'matrix' based on BMI and waist circumference. BMJ open, [s.I], v. 6, n. 3, p. 1-7, 2016. Disponível em: https://bmjopen.bmj.com/content/bmjopen/6/3/ e010159.full.pdf. Acesso em: 06 Mai. 2020.

BRASIL. Ministério da Saúde. Secretaria de Atenção à Saúde. Departamento de Atenção Básica. Contribuições dos núcleos de apoio à saúde da família para a atenção nutricional, Brasília, 2017. Disponível em: https://bvsms.saude.gov.br/bvs/publicacoes/contribuicoes_saude_ familia_atencao_nutricional.pdf. Acesso em: 25 Mar. 2020.

BRASIL. Ministério da Saúde. Secretaria de Vigilância em Saúde. Departamento de Vigilância de Doenças e Agravos não Transmissíveis e Promoção da Saúde. VIGITEL. BRASIL 2017. Vigilância de fatores de risco e proteção para doenças crônicas por inquérito telefônico: estimativas sobre frequência e distribuição sociodemográfica de fatores de risco e proteção para doenças crônicas nas capitais dos 26 estados brasileiros e no Distrito Federal em 2017, Brasília: Ministério da Saúde, 2018. Disponível em: https://bvsms.saude.gov.br/bvs/publicacoes/vigitel_ brasil_2017_vigilancia_fatores_riscos.pdf. Acesso em: 19 mar 2020.

BROWNING, L. M.; HSIEH, S. D.; ASHWELL, M. A systematic review of waist-to-height ratio as a screening tool for the prediction of cardiovascular disease and diabetes: 0.5 could be a suitable global boundary value. Nutr. Res. Rev., Cambridge, v. 23, n. 2, p. 247-269, 2010.

CENTER FOR DISEASE CONTROL. National Health and Nutrition Examiaion Survey. Anthropometry Procedures Manual. Processing. 2009. Disponível em: https://www.cdc.gov/nchs/data/nhanes/nhanes_07_08/ manual_an.pdf. Acesso em: 12 nov 2020

CERHAN, J. R. et al. A pooled analysis of waist circumference and mortality in 650,000 adults. Mayo Clin. Proc., Rochester, v. 89, n. 3, p. 335-345, 2014.

CORRÊA, M. M. et al. Waist-to-height ratio as an anthropometric marker of overweight in elderly brazilians. Cad. Saúde Pública, Rio de Janeiro, v. 33, n. 5, p. 1-14, 2017.
CORRÊA, M. M. et al. Habilidade da razão cintura-estatura na identificação de risco à saúde. Rev. Saúde Pública, São Paulo, v. 53, n. 66, p. 1-12, 2019.

DELBIM, L. R.; BACIUK, É. P. Avaliação de obesidade e sobrepeso como estratégia de diagnóstico organizacional para risco ocupacional: um estudo com motoristas de ônibus. Rev. Ciênc. Méd. Biol., Salvador, v. 15, n. 2, p. 178-185, 2016.

DESPRÉS, J. P. Body fat distribution and risk of cardiovascular disease: an update. Circulation, Dallas, v. 4, n. 126, p. 1301-13, 2012.

FERREIRA, A. P. DE S.; SZWARCWALD, C. L.; DAMACENA, G. N. Prevalência e fatores associados da obesidade na população brasileira: estudo com dados aferidos da Pesquisa Nacional de Saúde, 2013. Rev. Bras. Epidemiol., São Paulo, v. 22, e190024, 2019.

FERREIRA, S. R. G. et al. Cardiometabolic diseases. Rev. Bras. Epidemiol., São Paulo, v. 21, supl. 2, p. e180008, 2018.

GIBSON, S. A.; ASHWELL, M. Non-overweight 'apples' have higher cardiometabolic risk factors than overweight 'pears': waist-to-height ratio is a better screening tool than $\mathrm{BMI}$ for blood levels of cholesterol and glycated haemoglobin. Obesity Facts, , [s.l.], v. 8, n. 139, supl. 1, p. 11-12, 2015.

HAUN, D. R.; PITANGA, F. J. G.; LESSA, I. Razão cintura/estatura comparado a outros indicadores antropométricos de obesidade como preditor de risco coronariano elevado. Rev. Assoc. Med. Bras., São Paulo, v. 55, n. 6, p. 705-711, 2009.

INSTITUTO BRASILEIRO DE GEOGRAFIA E ESTATÍSTICA (IBGE). Pesquisa nacional de saúde 2013. Acesso e utilização dos serviços de saúde, acidentes e violências: Brasil, grandes regiões e unidades da federação. Rio de Janeiro, 2015. V. 39. Disponível em: https://biblioteca.ibge.gov. br/visualizacao/livros/liv94074.pdf. Acesso em: 25 Mar. 2020.

KLAUCK, C. M. et al. Comorbidades associadas à obesidade em pacientes candidatos à cirurgia bariátrica. RBONE, São Paulo, v.13, n. 79, p. 351-356, 2019.

LEVORATO, C. D. et al. Fatores associados à procura por serviços de saúde numa perspectiva relacional de gênero. Cien. Saúde Colet., Rio de Janeiro, v. 19, n. 4, p. 1263-1274, 2014.

MALTA, D.; MORAIS NETO, O.; SILVA JUNIOR, J. Apresentação do plano de ações estratégicas para o enfrentamento das doenças crônicas não transmissíveis no Brasil, 2011 a 2022. Epidemiol. Serv. Saúde, Brasília, v. 20 , n. $4,425-438,2011$.

MARTINS-SILVA, T. et al. Obesidade geral e abdominal em adultos residentes em zona rural no sul do Brasil. Rev. Saúde Pública, São Paulo, v. 52, supl. 1, p. 7s, 2018.

MILAGRES, L. C. et al. Waist-to-height ratio and the conicity index are associated to cardiometabolic risk factors in the elderly population. Cien. Saúde Colet., Rio de Janeiro, v. 24, n. 4, p. 1451-1461, 2019.

NATIONAL INSITUTE FOR HEALTH AND CARE EXCELLENCE. Obesity: identification, assessment and management. Nov. 2014. Disponível em: https://www.nice.org.uk/guidance/cg189. Acesso em: 19 Mar. 2020.

NEELAND, I. J. et al. Visceral and ectopic fat, atherosclerosis, and cardiometabolic disease: a position statement. Lancet Diabetes Endocrinol., Inglaterra, v. 7, n. 9, p. 715-725, 2019.

PITANGA, F. J. G.; LESSA I. Indicadores antropométricos de obesidade como instrumento de triagem para risco coronariano elevado em adultos na cidade de Salvador - Bahia. Arq. Bras. Cardiol., São Paulo, v. 85, n. 1, p.26-31, 2005. 
POIRIER, P. The many paradoxes of our modern world: is there really an obesity paradox or is it only a matter of adiposity assessment? Ann. Intern. Med., Philadelphia, n. 163, p. 880-881, 2015.

REIS, G. M. S. et al. Razão cintura/estatura e indicadores antropométricos de adiposidade. Braspen J., [s.I], v. 33, n. 4, p. 435-439, 2018.

REZENDE, A. C. et al. Is waist-to-height ratio the best predictive indicator of hypertension incidence? A cohort study. BMC public health, London, v. 18 , n. 281 , p. $1-11,2018$.

ROSS, R. et al. Waist circumference as a vital sign in clinical practice: a consensus statement from the IAS and ICCR working group on visceral obesity. Nat. Rev. Endocrinol., London, v. 16, n. 3, p. 177-189, 2020.
SAHAKYAN, K. R. et al. Normal-weight central obesity: implications for total and cardiovascular mortality. Ann. Intern. Med., Philadelphia, v. 163, n. 11, p. 827-835, 2015.

WANG, J. et al. Comparisons of waist circumferences measured at 4 sites. American Journal of Clinical Nutrition, New York, v. 77, n. 2, p. 379-384, 2003.

WORLD HEALTH ORGANIZATION. Obesity: preventing and managing the global epidemic: report of a WHO consultation. World Health Organization, 2000. (Technical Report Series, 894). Disponível em: https://www.who.int/nutrition/publications/obesity/WHO_TRS_894/ en/. Acesso em: 19 Mar. 2020.

Submetido em: $30 / 07 / 2020$

Aceito em: 02/03/2021 